\title{
KNOWLEDGE-BASED FUZZY INFERENCE SYSTEM FOR SEPSIS DiAgNOSIS
}

\author{
Igodan Charles Efosa ${ }^{1}$ and Akwukwuma V.V.N ${ }^{2}$ \\ Department of Computer Science, Faculty of Physical Sciences, University of Benin, \\ P.O. Box 1154, Edo State, Nigeria. \\ ${ }^{1}$ charlesigodan@ [yahoo,gmail].com; +2348062149776; ${ }^{2}$ vakwukwuma@yahoo.com; \\ $+2348033440003$
}

\begin{abstract}
In this study, a Fuzzy Inference System is developed to create a knowledge-based for the diagnosis and detection of sepsis using Matlab's fuzzy logic toolbox. The FIS consists of expert-specified input membership functions, output membership function, and IF-THEN rules. To validate our system, we tested it with the domain (medical) expert knowledge by comparing its performance with at least 10 hypothetical scenarios. The KB-FIS gave good answers in 8/10 scenarios and generated rules for decision support. The answers are qualitatively correct but quantitatively small. The fuzzy inference system designed performs as well as the medical experts under controlled conditions. With the methodology used in this study, it is hoped that it can assist in enhancing the knowledge-base system for proper, effective and early diagnosis and detection of sepsis
\end{abstract}

\section{KEYWORDS}

Fuzzy Inference System, Fuzzy Set Theory, sepsis, Knowledge-Based System, Neonatal.

\section{INTRODUCTION AND BACKGROUND}

The University of Benin Teaching Hospital (UBTH) is a Federal hospital in Edo State of Nigeria that runs the Neonatal Intensive Care Unit (NICU). The attendant problems in caring for infants in their early stage in life, in spite of equipments and staff who have specialized training in newborn care, are enormous. Babies sent into the NICU could: be born prematurely, have difficulties during deliveries, and show signs of problem in the first four weeks of life. Although not all babies in the NICU have the same illness or condition [11], some diagnoses carried out on babies include: Sepsis, Hypoxic ischemic encephalopathy (HIE), Asphyxia, Syndrome of inappropriate antidiuretic hormone secretion (SIADH), Meningitis, Pneumonia, Respiratory distress syndrome, Meconium aspiration syndrome, congestive heart failure, Necrotizing enterocolitis, Acute renal failure (ARF) to mention but a few. This study focuses on sepsis being one of the illnesses of newborn caused by bacteria that is found in the blood. According to [14], Neonatal sepsis is a clinical syndrome of bacteremia with systemic signs and symptoms of infection in the first 4 weeks of life. Sepsis is when the immune system responds to a serious infection by attacking the body's own organs and tissues. Sepsis causes the body's normal reaction to infection to go into overdrive. Bacteria from the infection and the toxins they create can change a person's body temperature, heart rate, and blood pressure, and prevent the body's organ from working properly [11]. Neonatal Sepsis can affect people of any age and according to DOI : $10.5121 /$ ijcsity.2013.1301 
[14], can be classified into two sub-types depending upon whether the onset of symptoms is before 72 hours of life (early onset) or later (late onset). [10], posit that in 1992, a consensus conference held by the American College of Chest Physicians decided that a patient will be diagnosed with sepsis if they present with Systemic Inflammatory Response Syndrome (SIRS), which is readily diagnosed by the presence of at least two of: fever or hypothermia, tachycardia, tachypnea or hyperventilation, leukocytosis or leucopenia in addition to a confirmed infection. However, due to the lack of specificity given by this list, the criteria were extended following an international consensus conference in 2001as [10]:

a. General variables: Fever or hypothermia, Tachypnea, Altered mental state, Unexplained hyperglycemia

b. Inflammatory variables: Leukocytosis or leucopenia, Increased CRP ( $>2 \mathrm{SD}$ above normal), Increased PCT (>2 SD above normal)

c. Tissue perfusion variables: Unexplained hyperlactatemia, Decreases capillary refill or skin mottling

d. Organ dysfunction variables: Unexplained hypoxemia, Acute oliguria, Coagulation abnormalities, Ileus, Hyperbilirubinemia, Thrombocytopenia

More specifically, severe sepsis is defined as sepsis with an associated acute organ failure, and septic shock is severe sepsis where the patient remains hypotensive despite adequate fluid resuscitation. These diagnoses do not define different illnesses, but instead define more serious conditions of the same illness. The uncertainty associated with the diagnosis of sepsis leads to the early demise of infants. These uncertainty, vagueness, and imprecision are very common in medicine where terms that are unavoidably vague are often used. Fuzzy Set Theory (FST), a powerful tool used to handle imprecision and uncertainty, can be used to deal with the concept of partial true and partial false values aiming at tractability, robustness and low cost-solutions for real world challenges. The benefits of using fuzzy set theory brings with it the attendant features and the ability to deal with linguistic terms which explains its adoption in solving problems in medicine and other areas of application. In [13], FST provides a systematic calculus to deal with information linguistically, and it performs numeric computation by using linguistic labels stipulated by membership functions. [1], opined that the theory of Fuzzy Sets has become an important mathematical approach in diagnosis systems; and, [4] it is recently being introduced into the epidemiology and public health section for solving health problems. The FST was introduced by Lotfi A. Zadeh in the 1960s as a means to model the uncertainty within natural language and introduced the concept of vagueness [13]. The manifestations of neonatal septicemia are often vague and therefore demand a high index of suspicion for early diagnosis. The study aimed at developing a knowledge-based system for decision support for addressing the bias and subjective judgment prevailing in human cognitive processes during early diagnosis of sepsis. The tool is in the form of a standardized inference system applying fuzzy logic knowledge-based fuzzy inference system (KBFIS), using MATLAB 7.10 proprietary Fuzzy Logic Module.

\section{BACKGROUND OF STUDY}

The application of Fuzzy Set Theory concept is predominant in medicine as well as other fields. Its application ranges from the diagnosis to therapy even as to common cough to a more severe illness. The ability to deal with vagueness, imprecision, and uncertainty of human natural language has made it pervasive. Here are some related literatures: In [2], a fuzzy linguistic model to evaluate the risk of neonatal death based on birth weight, gestation age, Apgar score and previous report of stillbirth was proposed. It aimed to build a computational predictive model using fuzzy logic. [8], developed an expert system, FLORENCE, for neonatal ventilation using the fuzzy logic toolkit from MATLAB 6 creating a multi fuzzy logic controller design. The input 
variables were defined as parameters that are critical for neonatal ventilation; whereas the output variables correlated with ventilator controls. [7], implemented a fuzzy controller for the adjustment of inspired oxygen concentration (FIO2) in ventilated newborns. The controller utilizes rules produced by neonatologists, and operates in real-time. Some majorly in the form of expert systems designed to give advice to clinicians, includes: VentPlan, a ventilator management advisor that interprets patient's physiologic data to predict the effect of proposed ventilator changes [6], KUSIVAR, a program designed as a comprehensive system for respiratory management during all phases of pulmonary disease [5], WeanPro, a program designed to help wean post-operatives patients from ventilators [9]. In spite of the best efforts of clinicians and researchers put into proffering solutions for early diagnosis and detection, sepsis still remains a leading cause of death in most critically ill patients the world over. It is clear from literature that although sepsis is a very well researched and documented subject within medical circles, it is often a very difficult condition to diagnose and treat [10]. This study intends to carry out the design of a knowledge-based Fuzzy Inference system for the diagnosis and detection of sepsis which is common among infants in their neonatal intensive care unit by stratifying patients according to risk using Fuzzy Logic based on linguistic descriptions. Unlike Boolean logic or classical logic, which assumes that every fact is either entirely true or false, fuzzy logic extends Boolean logic to handle vague and imprecise expressions. According to [12], the essential characteristics of fuzzy logic are: exact reasoning is viewed as a limiting case of approximate reasoning, everything is a matter of degree, any logic system can be fuzzified, knowledge is interpreted as a collection of equivalent and fuzzy constraints on a collection of variables, and inference is viewed as a process of propagation of fuzzy constraints. For more on fuzzy logic, inquisitive readers are referred to [12].

\section{METHOD}

Information on the salient symptoms of sepsis was gathered through interaction with the medical experts from the University of Benin Teaching Hospital and categorized. Neonatal sepsis is classified into the following categories: No Sepsis, At Risk, and Severe Sepsis. Data mining technique was applied using normalization method. The data mining technique, Min-Max and zscore methods, was used to normalize the variables between the range [ $\left.\begin{array}{ll}0 & 1\end{array}\right]$ before applying the Fuzzy inference system. The fuzzy inference system involves eleven (11) inputs from the general list which were carefully selected from the medical expert and useful articles from the Internet. Fuzzy inference systems are essentially knowledge-based systems utilizing Fuzzy logic, Fuzzy IF-THEN rules and Membership Functions, and is made up of five functional blocks as shown in Figure 1: (Noor 2004)

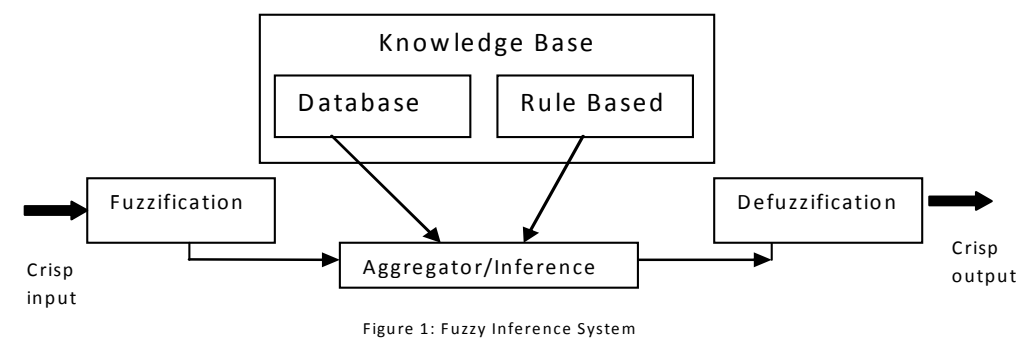

The steps performed by the fuzzy inference systems are outlined as:

a) Fuzzification Step: Comparison of the input variables with the membership functions of the premise part in obtaining the membership values of each linguistic term. 
b) Combination of the membership values of the premise part to deduce firing strength of each rule using the selected operator.

c) Generation of the consequence or results of each rule

d) Defuzzification Step: Aggregation of the results or consequences to produce a crisp.

The Fuzzy if-then rule assumes the form: IF $x_{i}$ is A THEN $y_{i}$ is B, where A and B are linguistic values defined by the fuzzy sets on universes of discourse $X$ and $Y$, respectively. Often $x_{i}$ is $A$ is called the antecedent or premise, while $y_{i}$ is $B$ is called the consequence or conclusion. In using the Mamdani's model, the inference process evaluates all rules in the rule base and combines the weighted consequents of all relevant rules into a single output fuzzy set [13]. In order to indicate an appropriate representative value for the final output, the aggregated output membership function has to be converted into a crisp form. The conversion is done using the Centroid or centre of Area (COA) computational schemes given in equation 1:

$$
Z_{C O A}=\frac{\int \mu_{A}(z) z d z}{\int \mu_{A}(z) d z}
$$

where $\mu_{A}(Z)$ is the aggregated output membership function. This is the most commonly used defuzzification technique and is very accurate. The only disadvantage is that it can be computationally difficult for complex membership functions [3].

\section{SYSTEM DESCRIPTION/RESULTS}

Fuzzy rule and fuzzy reasoning are the backbone of fuzzy inference systems, which are the most important modeling tool based on fuzzy set theory [13]. The logic of these inference rules are based on the expert knowledge of the neonatologists. The IF THEN Rules used are shown in Table 1:

Table 1. IF THEN Rules.

\begin{tabular}{|l|l|}
\hline $\begin{array}{l}\text { Rule } \\
\text { No }\end{array}$ & \multicolumn{1}{|c|}{ Conditions } \\
\hline 1 & $\begin{array}{l}\text { If (Albumin_level is Normal) and (Temp is Normal) and (CRP is Normal) and (Creatine is } \\
\text { Moderate) and (Lactate is Normal) and (Leukocyte is Normal) and (SBP is dNormal) and } \\
\text { (DBP is Normal) and (SaO2 is Normal) and (Heart_Rate is Normal) and (Resp_Rate is } \\
\text { Normal) then (Result is Not_Sepsis) (1) }\end{array}$ \\
\hline 2 & $\begin{array}{l}\text { If (Albumin_level is Low) and (Temp is Low) and (CRP is Normal) and (Creatine is Low) } \\
\text { and (Lactate is Low) and (Leukocyte is Low) and (SBP is Normal) and (DBP is Normal) } \\
\text { and (SaO2 is Low) and (Heart_Rate is Low) and (Resp_Rate is Low) then (Result is } \\
\text { No_Sepsis) (1) }\end{array}$ \\
\hline 3 & $\begin{array}{l}\text { If (Albumin_level is High) and (Temp is High) and (CRP is Abnormal) and (Creatine is } \\
\text { High) and (Lactate is High) and (Leukocyte is Abnormal) and (SBP is Prehypertension) } \\
\text { and (DBP is Prehypertension) and (SaO2 is High) and (Heart_Rate is High) and } \\
\text { (Resp_Rate is High) then (Result is Severe_Sepsis) (1) }\end{array}$ \\
\hline 4 & $\begin{array}{l}\text { If (Albumin_level is High) and (Temp is High) and (CRP is Abnormal) and (Creatine is } \\
\text { High) and (Lactate is High) and (Leukocyte is Abnormal) and (SBP is Hypertension) and } \\
\text { (DBP is Hypertension) and (SaO2 is High) and (Heart_Rate is High) and (Resp_Rate is } \\
\text { High) then (Result is Severe_Sepsis) (1) }\end{array}$ \\
\hline 5 & $\begin{array}{l}\text { If (Albumin_level is High) and (Temp is High) and (CRP is Abnormal) and (Creatine is } \\
\text { High) and (Lactate is High) and (Leukocyte is Abnormal) and (SBP is Hypertension) and } \\
\text { (DBP is Prehypertension) and (SaO2 is High) and (Heart_Rate is High) and (Resp_Rate is } \\
\text { High) then (Result is Severe_Sepsis) (1) }\end{array}$ \\
\hline
\end{tabular}


The AND operator used in the rules infers that the minimum criterion is used in the resultant and aggregated to indicate the condition of sepsis - No Sepsis, At Risk, Sepsis, and Severe.

Figure 2 shows the Fuzzy inference system while Figures 3 and 4 show the input and output membership functions for a no sepsis condition and a severe sepsis condition respectively.

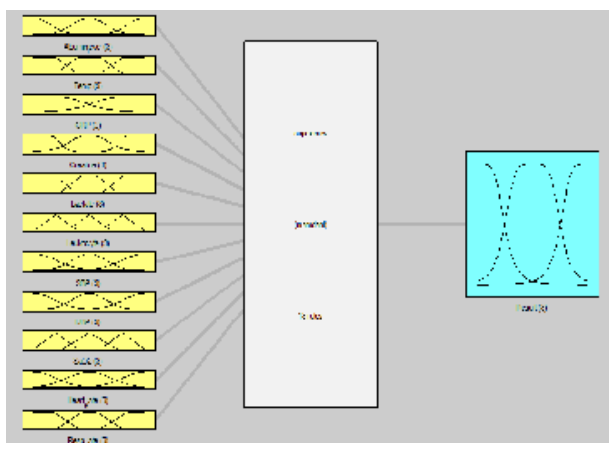

Figure 2. Fuzzy Inference System - sepsis

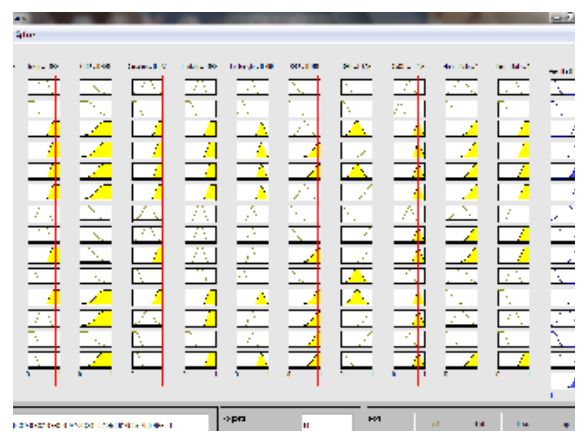

Figure 4. Fuzzy Inference System - sepsis

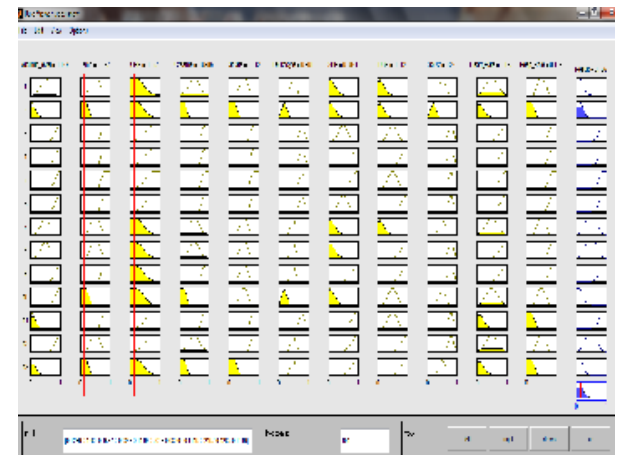

Figure 3. Fuzzy Inference System - No sepsis

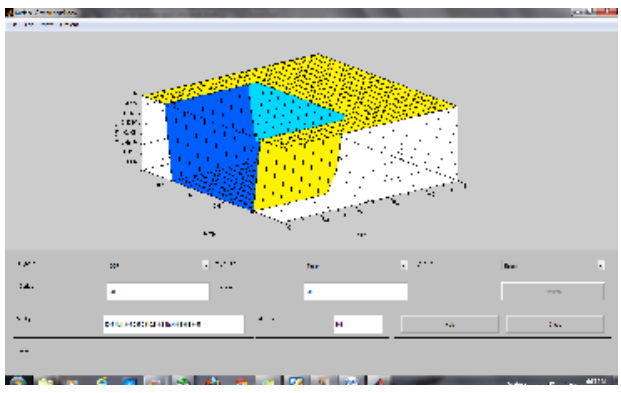

Figure 5. A 3-dimensional plot of Sepsis FIS showing the relationship between the two inputs and output

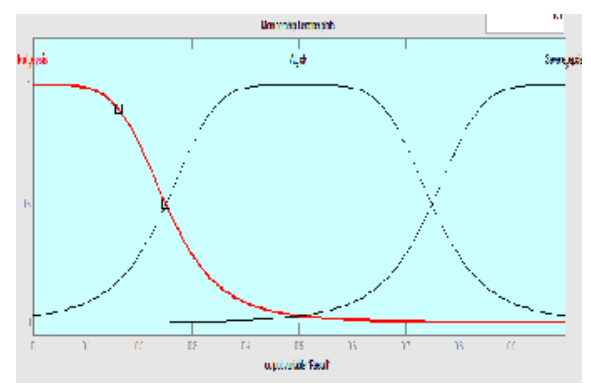

Figure 6. Output variable with 3 Membership Functions

Combining both the input membership functions and the output membership function with the rules, a 3-dimensional plot can be obtained to give a snapshot relationship between the inputs and output of the FIS as shown in Figure 5 above while the output membership function is shown in Figure 6 above. 


\section{DISCUSSION}

The designed Knowledge-based Fuzzy Inference System was tested with the expert's knowledge to ascertain its effectiveness and got positive answers. We are concluding that it will be of immense benefit as a knowledge-based decision support to the medical doctors because of the variability of the disease condition. The heart of the neonatal sepsis KB-FIS is the knowledge base that forms the Inference Engine. In this case, the knowledge of the expert is translated to Fuzzy Rules (IF-THEN Rules) used in the Inference Engine. It might be argued that the level of expertise from one human expert may differ from another such that one expert's design of the rules may not be the same with another, but as long as they are fixed in the Inference Engine, the output of the FIS may always be consistent. In this regard, it is possible to add useful rules or delete unwanted rules where necessary. It has been shown that the FIS works by converting qualitative data of the patient's health status to numeric values; that makes it possible to quantify the level of infection. To validate our system, we tested it with the domain (medical) expert knowledge by comparing its performance with at least 10 hypothetical scenarios. The KB-FIS gave good answers in 8/10 scenarios and generated rules for decision support. The answers are qualitatively correct but quantitatively small. The fuzzy inference system designed performs as well as the medical experts under controlled conditions. It is hoped that the methodology adapted in this study will assist in creating a knowledge-based system for the diagnosis of sepsis. We would like to state clearly, that Neonatal sepsis is the single most important cause of neonatal deaths. If diagnosed early and treated aggressively with antibiotics and good supportive care, it is possible to save most death cases of neonatal sepsis in the NICU.

\section{Conclusion}

There are, however, some noticeable limitations we suspect in the FIS, they include:

a. The use of descriptive knowledge may not be as effective as physical appearance of the patients

b. The output of the FIS is restricted by the number of membership functions and rules of the knowledge base in the Inference Engine.

c. Worst of all is our inability to include other causative factors of neonatal sepsis.

In our study, we discovered that the specification of an appropriate membership function and the identification of a suitable universe of discourse aid in the construction of Fuzzy Set. And most cases the specification of an appropriate membership function is more or less subjective. In the nearest future, we intend to apply the combination of neural network and Fuzzy Logic for learning and adaptation. The sensitivity of the knowledge-based FIS will be looked into by means of adding more rules to the Fuzzy Inference Engine.

\section{REFERENCES}

[1] Adlassnig, K-P (2000) Proceedings of Erudit Workshop Fuzzy Diagnostic and Therapeutic Decision Sup-port. Vienna: Österreichishe Computer Gessell-schaft.

[2] Fernando, L., Nascimento, C., Paloma, M., Rizol, R and Abiuzl, L (2009) "Establishing the risk of neonatal mortality using a fuzzy predictive model”, Cad Saude Publicam Rio de Janeiro, 25(9): 2043-2052.

[3] Noor, M.J.M. (2004). Application of knowledge-based fuzzy inference system on high voltage transmission line maintenance. A thesis submitted for the degree of master of engineering.

[4] Ortega, N.R.S. Sallum, P.C. Massad, E. (2000) "Fuzzy dynami-cal system in epidemic modeling", Kybernetes; 29:201-18. 
[5] Rudowski, R., Frostell, C and Gill, H. (1989) “A Knowledge-based support system for mechanical ventilation of the lungs", The KUSIVAR concept and prototype. Comput Methods Programs Biomed, 30, 59.

[6] Rutledge, G and Thomson, G (1991) "Ventplan: a Ventilator-Management Advisor", Proc. Annu Symp Comput Appl Med Care, 869.

[7] Sun, Y. Kohane, I. and Stark, A.R. "Fuzzy Logic Assisted Control of Inspired Oxygen in Ventilated Newborn Infants”, AMIA, Inc. 0195-4210 (1994).

[8] Tan, T., Snowden, C. Evans, Baxter, G. and Brownlee, K.G (2013) "Fuzzy Logic Expert System for Neonatal Ventilation”, Journal of Medical Engineering \& Technology. 21(2): 67-73.

[9] Tong, D (1991) "Weaning patients from mechanical ventilation. A Knowledge-Based system approach. Comput Methods Programs Biomed, 38, 267.

[10] Ward, L. (2012) Application and Testing of Modifications to the TREAT Sepsis Networks.

[11] http://kidshealth.org/parent/system/ill/nicu_diagnoses.html. February 2013.

[12] Zadeh, A.L. (1965) "Fuzzy Set", Information and Control, Vol. 8, pp. 338-358.

[13] Jang, J.-S.R., Sun, T.C., Muzitani, E. (1997) "Neuro-Fuzzy and Soft-Computing. Computational Approach to Learning and Machine Intelligence." Prentice Hall, Inc.

[14] Mehta, K., Bhatta, N.K., Majhi, S., Shrivastava, M.K. and Sing, R.R. (2012) "Role o/f Zinc In Neonatal Sepsis: A Double Blinded, Randomized, Placebo Controlled Trial” Indian Pediatrics. Available from: URL: http://www.indianpediatrics.net/epub05.10.12/RP-127.pdf. Accessed 7th of July, 2013.

\section{Authors}

Igodan Charles Efosa, is a lecturer in the Department of Computer Science, University of Benin, Edo State, Nigeria. He has obtained his B.Sc. and Masters' degree in Computer Science. He is currently working towards his $\mathrm{Ph} . \mathrm{D}$. degree in machine learning. He has worked as system engineer/consultant and programme analyst for eight years and has taught in various institutions. His interests are in Data mining, knowledge warehousing, Expert system, and Soft-Computing and Machine Learning.

Dr. (Mrs.) V.V.N. Akwukwuma is currently with the Department of Computer Science, Faculty of Physical Sciences, University of Benin, Nigeria. Her research has focused on Software Engineering particularly in the areas of Software Metrics and Software security. She is currently a member of MAN, CPN, NCS, INWES and OWSD. She can be reached by phone on +2348033440003 and email vakwukwuma@ yahoo.com.
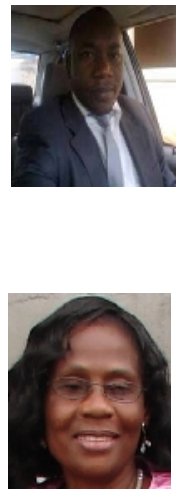